\title{
Pharyngeal-Cervical Brachial Variant of Guillain-Barré syndrome mistaken for a case of Surgical Dysphagia
}

\author{
Megan Marshal ${ }^{1}$ \\ ${ }^{1}$ Beaumont Hospital
}

September 25, 2021

\begin{abstract}
We present the case of a 41 year old gentleman who was admitted to hospital with a presumed case of surgical dysphagia. A clinical diagnosis of pharyngeal-cervical-brachial variant of Guillain-Barré Syndrome was made and he treated with a 5 day course of IVIG resulting in gradual but signigicant symptomatic relief.
\end{abstract}

\section{Introduction}

The PCB variant of GBS is characterised by the progressive development of oropharyngeal, neck and shoulder muscle weakness with associated dysphagia. ${ }^{1}$ It may be associated with facial weakness. Lower limb strength and reflexes are generally preserved. ${ }^{1}$ Nagashima et $\mathrm{al}^{2}$ maintain Ropper's original description of "pure PCB" as a patient that presents with rapidly progressive oropharyngeal and cervicobrachial weakness, associated with hyporeflexia or areflexia in the absence of ophthalmoplegia or leg weakness. ${ }^{3}$ Notably, antecedent infectious symptoms are often present and form a key component of the pathophysiological mechanism of GBS as a whole. This case represents an intersection between surgical and medical specialities. Our patient was initially admitted as a case of surgical dysphagia and investigated as such. However, a delay in diagnosis arose as the initial investigations were undertaken with a surgical diagnosis in mind This case illustrates the possible disciplinary overlap that occurs in presentations of dysphagia in which a neurological cause may not be considered within the differential diagnosis.

\section{Case Report}

A 41 year old gentleman was admitted under the surgical team with a two week history of dysphagia to both solids and liquids associated with regurgitation and dysphonia. On initial examination, he was apyrexic and vitally stable. Admitted under the surgical team he had no neurological examination performed at the time of initial presentation. Of note, his BMI was significantly reduced as the patient reported over $14 \mathrm{lbs}$ of weight loss over the previous two weeks, secondary to reduced intake.

Initial bloodwork revealed elevated ESR, WCC, CRP and CPK. Gastroscopy demonstrated no findings to indicate an obstructive cause for dysphagia. Following review by the ENT team, infectious causes such as epiglottitis were excluded but left vocal cord paralysis was noted. Recommendation was made for CT of the neck and thorax, full medical work up and speech and language assessment. It was at this point that a neurology opinion was sought.

Focussed history revealed recent flu-like illness two weeks prior to the onset of symptoms. Neurological examination revealed symmetrical upper limb weakness. Specifically, weakness of shoulder abduction with mild symmetric hyperreflexia with some subtle dysmetria in the upper limbs. Sensation and tone were intact with no abnormalities of gait. Differentials at this stage included brainstem pathology, postinfectious or inflammatory neuropathy, structural disease and demyelination. 
Initial work-up involved MRI brain and cervical spine, lumbar puncture, serum viral screen, connective tissue disease screen, haematinics and anti-ganglioside antibodies. Of note, MRI brain and cervical spine demonstrated no parenchymal enhancement, no evidence of brainstem abnormality and no findings supportive of demyelination. Lumbar demonstrated albumin-cytological dissociation with an otherwise normal cell count and glucose. This gentleman's case was subsequently discussed at the neurology multidisciplinary meeting and it was agreed that the most likely diagnosis was some form of post-infectious, immune mediated inflammatory neuropathy given the antecedent respiratory symptoms, the albumin-cytologic dissociation and an elevated serum IgG and IgM. The patient was commenced on a five day course of intravenous immunoglobulin (IVIG). By day five, the patient felt subjectively better with an improvement in dysphonia and tolerance for oral fluids without regurgitation.

Given the patient's initial presentation and his response to IVIG, a clinical diagnosis of PCB variant of GBS was made. The efficacy of IVIG in the treatment of GBS and its immune mediated neuropathies is well documented. ${ }^{4}$ In some cases, electromyography may have supported this diagnosis, specifically looking for $\mathrm{F}$ wave prolongation or any evidence that would support demyelination. However, nerve conduction studies were not available in our facility. Furthermore, this diagnosis was made while the patient's antiganglioside antibody results were still pending. Initial video-fluoroscopy (VFS) demonstrated moderate to severe pharyngeal dysphagia. Subsequent VFS two weeks later was significantly improved and reported as mild to moderate dysphagia. Ten days post-IVIG completion, the patient continued to make steady progress and was discharged home on a modified consistency diet.

\section{Discussion}

Guillain Barre Syndrome is a well-recognised syndrome, estimated to result from an immune mediated response to an antecedent infection. ${ }^{5}$ Pathophysiological mechanisms include cross-reaction of antibodies with peripheral nerve components, resulting in either demyelinating or axonal forms of the disease. ${ }^{5}$ As illustrated by nerve conduction studies, the particular mechanism of PCB is axonal rather than demyelinating and is supported by decreased distal motor and/or sensory amplitudes. ${ }^{6}$ Based on serial conduction studies PCB is understood to be a regional variant of GBS, representing a form of acute motor axonal neuropathy (AMAN) as opposed to Acute inflammatory demyelinating polyneuropathy (AIDP). ${ }^{1}$

It is well documented that approximately $10 \%$ of patients present with normoreflexia or hyperreflexia as was the case with our patient. ${ }^{1}$ These patients have been labelled as PCB with preservation of muscle stretch reflexes. ${ }^{1}$ Autoantibodies against specific neuronal gangliosides are the proposed mechanism of action for AMAN as previously mentioned. Susuki et al suggest that the binding of autoantibodies likely results in the activation of the complement cascade and the inhibition of voltage gated sodium channels, resulting in the disruption of neuromuscular junctions. ${ }^{7}$ The strongest association in PCB is the presence of IgG anti-GT1a antibodies[5]. However, many cases are also associated with anti-Gq1b and anti-GM1 antibodies. ${ }^{5}$ Notably, all tested anti-ganglioside antibodies for our patient were negative. However, the finding was only noted weeks after the patient was discharged. It has been stated in the most recent diagnostic criteria for PCB that antiganglioside antibodies are diagnostically supportive but are not definitively required for diagnosis. ${ }^{1}$ This diagnostic criteria for PCB, as set out by Wakerly and Yuki, designates the features required for diagnosis and those that are strongly supportive of the diagnosis. ${ }^{1}$ Relatively symmetric oropharyngeal weakness with neck and arm weakness, along with arm areflexia or hyporeflexia is required, along with the absence of ataxia, disturbed consciousness or leg weakness. Furthermore, the illness pattern should be monophasic with an interval between onset and symptomatic peak of 12 hours to 28 days with a subsequent clinical plateau. Features such as preceding infectious symptoms, CSF albumin-cytological dissociation, EMG evidence of neuropathy and positive IgG anti-GT1a or anti-GQ1b antibodies are solely supportive of a diagnosis of PCB variant of GBS. ${ }^{1}$

\section{Conclusion}

This case illustrates a classical presentation of a relatively uncommon neurological illness within a surgical setting. A high index of clinical suspicion is required in cases presenting with acute or subacute dysphagia. 
Differential diagnosis should incorporate both surgical and medical causes, with a low threshold for seeking specialist opinion. The risk of respiratory compromise in neurological causes of dysphagia means that delayed diagnosis may have devastating consequences on patient survival. As such, a presentation of dysphagia warrants thorough neurological examination in the first instance, along with exclusion of surgical causes. Any abnormality of which should trigger the physician or surgeon to seek alternative specialist opinion and advice.

\section{Acknowledgements}

None.

\section{Conflict of Interest}

No conflicts of interest.

\section{Funding}

There was no source of funding for this case report.

\section{Ethical Approval}

No ethical approval was required.

\section{Consent}

Patient consent was received.

\section{Guarantor}

Dr Megan Marshal.

\section{References}

1. Wakerley BR, Yuki N. "Pharyngeal-cervical-brachial variant of Guillain-Barre syndrome." J Neurol Neurosurg Psychiatry . 2014 Mar;85(3):339-44. doi: 10.1136/jnnp-2013-305397. Epub 2013 Jun 26.

2. Nagashima T, Koga M, Odaka M, et al. "Continuous spectrum of pharyngeal-cervical-brachial variant of Guillain-Barré syndrome." Arch Neurol 2007;64:1519-23.

3. Ropper AH. "Unusual Clinical Variants and Signs in Guillain-Barré Syndrome." Arch Neurol . 1986;43(11):1150-1152. doi:10.1001/archneur.1986.00520110044012

4. Yuki N, Hartung HP. "Guillain-Barré syndrome." N Engl J Med2012;366: 2294-304.

5. Vriesendorp, F. 2018. "Guillain-Barré Syndrome In Adults: Clinical Features And Diagnosis." [online] uptodate.com; accessed 1 March 2020.

6. Hadden RD, Cornblath DR, Hughes RA, Zielasek J, Hartung HP, Toyka KV, Swan AV. "Electrophysiological classification of Guillain-Barré Syndrome: clinical associations and outcome. Plasma Exchange/Sandoglobulin Guillain-Barré Syndrome Trial Group." Ann Neurol . 1998 Nov;44(5):780-8.

7. Susuki K, Rasband MN, Tohyama K, et al. "Anti-GM1 antibodies cause complement-mediated disruption of sodium channel clusters in peripheral motor nerve fibers." J Neurosci 2007;27:3956-67; McGonigal R, Rowan EG, Greenshields KN, et al. "Anti-GD1a antibodies activate complement and calpain to injure distal motor nodes of Ranvier in mice." Brain 2010;133:1944-60. 